\title{
Nonlinear Fiber Bragg Grating Based Optical Switching and Transistor Characteristics
}

\author{
Poornima Rawat ${ }^{1 *}$, Santosh Pawar ${ }^{2}$ and Tryambak Hiwarkar ${ }^{1}$ \\ ${ }^{1}$ Dept. of Electronics \& Communication, Sri Satya Sai University of Technology \& Medical Sciences, Sehore (M.P.), India \\ ${ }^{2}$ Dept. of Electronics \& Communication, Dr. A. P. J. Abdul Kalam University, Indore (M.P.), India \\ *Corresponding author_Email: poornima_rawat@yahoo.com
}

Available online at: www.isroset.org

Received: 10/Jan/2019, Accepted: 06/Feb/2019, Online: 28/Feb/2019

\begin{abstract}
The present research work provides a very simple analytical analysis of optical switching and transistor characteristics of nonlinear fiber Bragg grating. The nonlinear coupled mode equations have been solved to analyze the wave propagation in grating that exhibit attractive nonlinear optical property that makes them suitable for all-optical communication system. We derived the solutions for both forward and backward propagating field amplitudes considering the propagation of a high intense beam. Using the expression of reflectivity derived under nonlinear regime we examine the nonlinear switching and optical transistor behavior of fiber Bragg grating. We also obtained the expression for peak reflectivity of the grating under nonlinear Kerr regime. Using these expressions we have studied the peak reflectivity as a function of grating length at different input intensities.
\end{abstract}

Keyword-Optical Switching, Optical Transistor, Peak Reflectivity, fiber Bragg Grating, Nonlinear Coupled Mode Equation

\section{INTRODUCTION}

The ever-increasing demand for faster and more reliable devices to process the optical signals offers new opportunities in developing all-optical signal processing systems. All-optical switches, all-optical memories, all-optical limiters, all-optical discriminators and all-optical transistors [1] are only a few of the many devices proposed during the last two decades. In particular, it has been suggested that electronic transistors may one day be replaced by all-optical transistors, which will perform all the operations that their electronic counterparts do: such as amplification, switching, modulation and detection. The all-optical transistor action was first observed in the context of optical bistability [1, 2] occurs in a strong differential gain regime, where, for small variations in the input intensity, the output intensity switches abruptly from its low value to high value.

In view of the fast switching nonlinear photonic devices, the use of fiber Bragg grating (FBG) structures deserve particular attention because of its compact structure [3-7]. Winful et al were first investigated the nonlinear properties of periodic media and showed the possibility of bistability, multistability and switching behavior [8]. De sterke et al. [9] investigated numerically the transient properties of periodic media and suggested that the periodic media exhibit bistable behavior as well as periodic and chaotic self oscillation at high excitation intensity. Wabnitz [5] studied numerically the bistable switching characteristics of optical pulses propagating through fiber Bragg grating and discussed the role of the input pulse time width, mean wavelength and peak power on the selfswitching, pulse compression and break-up of the transmitted and reflected pulses. Lee and Agrawal [4] considered both the uniform and phase shifted grating and compared their performance numerically as a nonlinear switch when optical pulses are sent to the grating. The experimental studies of Melloni [10] and Taverner [11] on bistable switching phenomena in FBG demonstrated the operation of all optical gates based on the formation and transmission of coupled gap solitons. Recently, Yosia et al. [12] have observed double optical bistability in nonlinear $\pi$-phase shifted chalcogenide fiber Bragg grating (c-FBG). Their numerical study suggests all optical transistor operation in such a device.

The present work deals with the analytical study of optical switching, optical transistor action and intensity dependent variation in peak reflectivity of fiber Bragg grating under nonlinear Kerr regime. Using nonlinear coupled mode equations, we have obtained the intensity dependent expression for reflectivity and peak reflectivity of fiber Bragg grating. The work in this paper summarizes as follows: In 
section II we describe the mathematical model to obtain the expression of nonlinear reflectivity and peak reflectivity. In Section III, The optical switching phenomena have been described by plotting the reflectivity as a function of input intensity. In Section IV the optical transistor characteristics of fiber Bragg grating has been examined. A intensity dependent peak reflectivity of FBG are presented in Section V. Using these expressions we have studied the peak reflectivity as a function of grating length at different input intensities

\section{MAThematical MODEL}

At sufficiently high input intensity the intensity dependent of RI of FBG can be written as

$$
n(\omega, z)=n_{\text {eff }}(\omega)+n_{2}|E(z)|^{2}+n_{g}(z)
$$

The wave propagation in nonlinear FBG is governed by following NLCMEs [2]

$$
\begin{array}{r}
i \frac{\partial A_{f}}{\partial z}+\delta A_{f}+\kappa A_{b}+\gamma\left(\left|A_{f}\right|^{2}+2\left|A_{b}\right|^{2}\right) A_{f}=0, \\
-i \frac{\partial A_{b}}{\partial z}+\delta A_{b}+\kappa A_{f}+\gamma\left(\left|A_{b}\right|^{2}+2\left|A_{f}\right|^{2}\right) A_{b}=0 .
\end{array}
$$

Here, $\delta, \kappa$ and $\gamma$ are detuning parameter, linear coupling coefficient and nonlinear parameter, respectively, and are defined as

$$
\begin{aligned}
& \delta=2 \pi n_{e f f}\left(\frac{1}{\lambda}-\frac{1}{\lambda_{B}}\right), \times=\frac{2 \pi n_{g}}{\lambda_{B}} \text { and } \\
& \gamma=\frac{2 \pi n_{2}}{\lambda_{B}} .
\end{aligned}
$$

In the following analysis we have solved the above NLCMEs analytically by neglecting higher order terms of backward propagating mode and solutions are obtained as [13]

$$
\begin{aligned}
& A_{f}(z)=A_{1} \exp (i S z)+A_{2} \exp (i T z), \\
& A_{b}(z)=B_{1} \exp (i S z)+B_{2} \exp (i T z) .
\end{aligned}
$$

with $S=\frac{-\gamma I_{0}+\sqrt{\gamma^{2} I_{0}^{2}+4 q_{n l}^{2}}}{2}$ and $T=\frac{-\gamma I_{0}-\sqrt{\gamma^{2} I_{0}{ }^{2}+4 q_{n l}^{2}}}{2}$.

Here, $\quad I_{0}=\left|A_{f}\right|^{2}+\left|A_{b}\right|^{2}$ is the input intensity at Bragg wavelength $\lambda_{\mathrm{B}} . \mathrm{q}_{\mathrm{nl}}$ is the nonlinear dispersion relation in cubic medium and is defined as

$$
q_{n l}= \pm \sqrt{q^{2}+\delta \gamma\left(I_{o}+2\left|A_{f}\right|^{2}\right)}
$$

where $q\left(=\left(\delta^{2}-\kappa^{2}\right)^{1 / 2}\right)$ being the linear dispersion parameter for the Bragg grating. On substituting parameter $\mathrm{q}_{\mathrm{nl}}$ in equations (5) and (6), the fields of forward and backward propagating modes take the form [13]

$$
A_{f}(z)=A_{1} \exp (i S z)+r_{n l} B_{2} \exp (i T z)
$$

and

$$
A_{b}(z)=B_{2} \exp (i T z)+r_{n l} A_{1} \exp (i S z) \text {. }
$$

Here, $r_{n l}$ is the effective reflection coefficient in nonlinear regime and using equation (6) in equation (8) and (9) it is found to be

$$
r_{n l}=-\frac{\kappa}{S+\delta+\gamma\left(I_{0}+\left|A_{f}\right|^{2}\right)} .
$$

Applying the boundary conditions that light is incident only at the front end at $\mathrm{z}=0$ of the FBG as shown in Figure1, the nonlinear reflection coefficient $\left(r_{n g}\right)$ for a grating of length $\mathrm{L}$ has been obtained by using equations (7) to (10) as

$$
r_{n g}=\frac{A_{b}(0)}{A_{f}(0)}=\frac{-\kappa \psi[1-\exp (i 2 \Phi)]}{\psi^{2}-\kappa^{2} \exp (i 2 \Phi)}
$$

where $\Phi=k L / 2, \Psi=\left(\frac{k}{2}+\Delta\right)$,

$\Delta=\delta+\gamma\left|A_{f}\right|^{2}$ and $k=\sqrt{\gamma^{2} I_{0}^{2}+4 q_{n l}^{2}}$.

The corresponding expression for the reflectivity $\boldsymbol{R}_{n g}\left(=\left|r_{n g}\right|^{2}\right)$ in the nonlinear regime is

$$
R_{n g}=\frac{4 \kappa^{2} \psi^{2} \sin ^{2}(\Phi)}{\left[\psi^{2}-\kappa^{2}\right]^{2}+4 \kappa^{2} \psi^{2} \sin ^{2}(\Phi)}
$$

At resonance there is no detuning i.e $\delta=0$, hence the reflectivity is maximum. The expression for the peak reflectivity becomes:

$$
R_{n g(M A X)}=\left|r_{n g}(\delta=0)\right|^{2}
$$

\section{Study Of Optical SWitching Phenomena In Fiber BRAgG GRATING}

The optical switching behavior of fiber Bragg grating in Kerr regime is studied by plotting the reflected intensity $\left(\mathrm{R}_{\mathrm{ng}} \times \mathrm{I}_{0}\right)$ as a function of input intensity in Figure 1 for two different incident wavelengths of $1550.6 \mathrm{~nm}$ (doted curve) and 1550.8 $\mathrm{nm}$ (solid curve), respectively. To study the switching behavior of nonlinear FBG, we have plotted its reflected intensity as a function input intensity in Figure 1 using Equation (12). The numerical estimates are made with following FBG parameters of chalcogenide glass; effective mode refractive index $\mathrm{n}_{\mathrm{eff}}=$ $2.45, \lambda_{B}=1550 \mathrm{~nm}$, induced refractive index change $n_{\mathrm{g}}=$ $1 \times 10^{-4}$ and $\mathrm{n}_{2}=2.7 \times 10^{-17} \mathrm{~m}^{2} / \mathrm{W}$. All the incident wavelengths considered above are lying outside of the stop band of the fiber Bragg grating. In linear case, these wavelengths are completely transmitted through the FBG and structure works in 
transmission mode. As the intensity of the input beam increases the stop band of the Bragg grating detunes itself towards the higher wavelength side from its original position (at $\lambda_{\mathrm{B}}$ ) because of the presence of the positive Kerr nonlinearity in the medium. If the input intensity is sufficiently high, the Bragg wavelength $\lambda_{\mathrm{B}}$ is no longer lies within the stop band and the wavelength $\lambda>\lambda_{\mathrm{B}}$ comes in the stop band as a result these wavelength get reflected. The intensity of reflected waves increases with increasing input intensity and reaches its maximum value at particular input intensity as seen in Figure 1

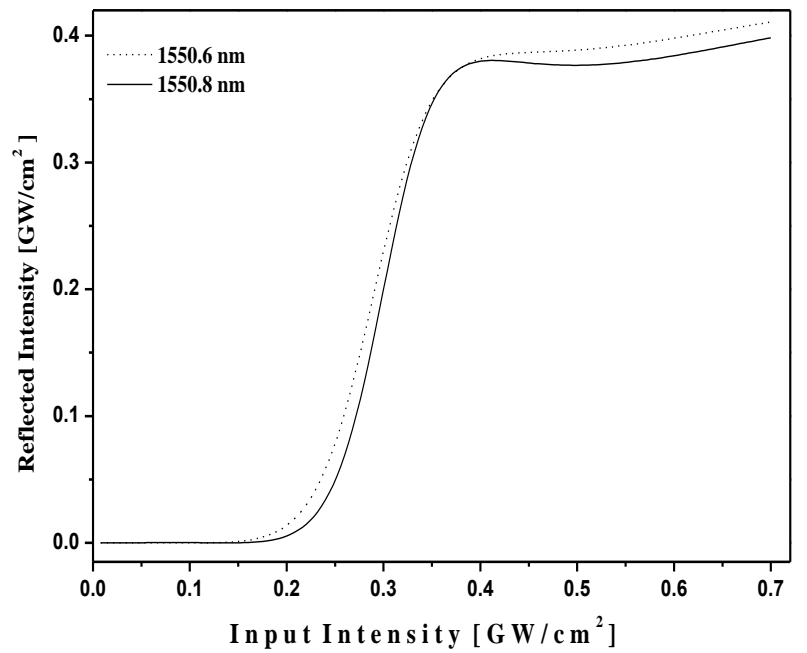

Figure 1: Optical switching action in nonlinear FBG two different incident wavelengths of $1550.6 \mathrm{~nm}$ (doted curve) and $1550.8 \mathrm{~nm}$ (solid curve), respectively.

It is found that the switch-on intensity is slightly increases with increasing the detuning wavelength. For the wavelength $1550.6 \mathrm{~nm}$ which is near the edge of the photonic bandgap the device requires low switch-on intensity $\mathrm{I}_{0} \approx 0.18 \mathrm{GW} / \mathrm{cm}^{2}$. When, the wavelength of the incident field detuned more and more away from the edge of the stop band the switch-on intensity increases as seen in Figure 1.

\section{STUDY OF OPTICAL Transistor ACTION IN NONLINEAR FIBER BRAGG GRATING}

We have discussed the optical transistor behavior of fiber Bragg grating in Kerr regime by plotting the reflected intensity $\left(\mathrm{R}_{\mathrm{ng}} \times \mathrm{I}_{0}\right)$ as a function of input intensity in Figure 2. The numerical estimates are made with following FBG parameters of chalcogenide glass; effective mode refractive index $n_{\text {eff }}=$ 2.45, Bragg grating design at wavelength $\lambda_{\mathrm{B}}=1550 \mathrm{~nm}$, nonlinear refractive index $n_{2}=2.7 \times 10^{-17} \mathrm{~m}^{2} / \mathrm{W}$, induced refractive index change $\mathrm{n}_{\mathrm{g}}=1 \times 10^{-4}$ and grating length as $5 \mathrm{~mm}$. We have chosen tunable quasi-CW laser source in $\mathrm{C}$-band (1535-1565 nm) and plots are obtained for three different incident wavelengths of $1550.5 \mathrm{~nm}$ (Fig. 2a), $1551 \mathrm{~nm}$ (Fig. 2b) and $1551.5 \mathrm{~nm}$ (Fig. 2c).

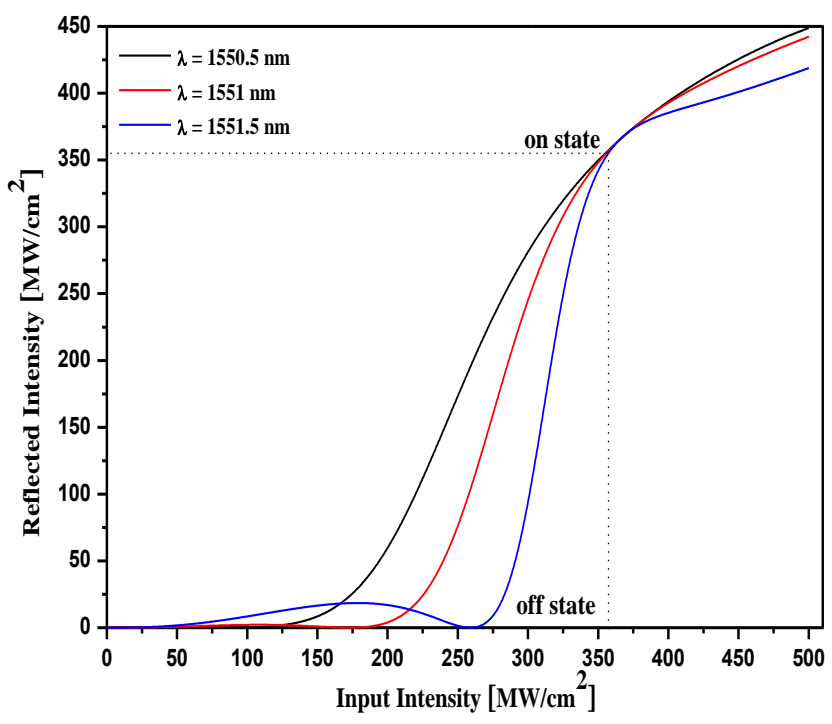

Figure 2: Optical transistor action in nonlinear FBG for three different incident wavelengths of $1550.5 \mathrm{~nm}, 1551 \mathrm{~nm}$ and $1551.5 \mathrm{~nm}$

All the incident wavelengths considered above are lying outside of the stop band of the fiber Bragg grating. In linear case, these wavelengths are completely transmitted through the FBG and structure works in cut off mode just like an electronic transistor. As the intensity of the input beam increases the stop band of the Bragg grating detunes itself towards the higher wavelength side from its original position (at $\lambda_{B}$ ) because of the presence of the positive Kerr nonlinearity in the medium. If the input intensity is sufficiently high, the Bragg wavelength $\lambda_{\mathrm{B}}$ is no longer lies within the stop band and the wavelength $\lambda>\lambda_{B}$ comes in the stop band as a result these wavelength get reflected. The intensity of reflected waves increases with increasing input intensity and reaches its maximum value at particular input intensity as seen in Figure 2. This is the saturation region of the optical transistor. It is found that the switch-on intensity of optical transistor increases with increasing the detuning wavelength. For the wavelength 1550.5 $\mathrm{nm}$ which is near the edge of the photonic bandgap the device requires low switch-on intensity $\mathrm{I}_{0} \approx 150 \mathrm{MW} / \mathrm{cm}^{2}$. When, the wavelength of the incident field detuned more and more away from the edge of the stop band the switch-on intensity increases as seen in Fig. 2. Hence, the bistable switching phenomenon of nonlinear fiber Bragg grating can be utilized in all optical transistor switching applications.

\section{Intensity DePendent PeAK ReFlectivity OF Fiber BRAgG GRATING}

We have discussed the peak reflectivity behavior of fiber Bragg grating in Kerr regime by plotting the peak reflectivity $R_{n g(M A X)}$ obtained in Equation (13) as a function of FBG length (L) at different input intensities in Figure 3. 


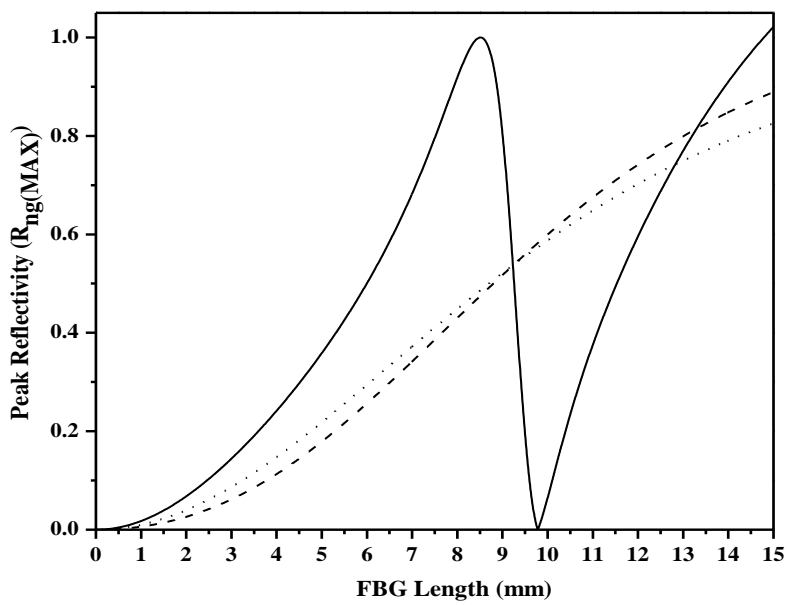

Figure 3: Peak reflectivity as a function of Bragg grating length (L), calculated for different values of input intensities (a) $1 \mathrm{MW} / \mathrm{cm}^{2}$ (dot curve), (b) $50 \mathrm{MW} / \mathrm{cm}^{2}$ (dashed curve) and (c) $100 \mathrm{MW} / \mathrm{cm}^{2}$ (solid curve).

The numerical estimates are made with following FBG parameters of chalcogenide glass; effective mode refractive index $n_{\text {eff }}=2.45$, Bragg grating design at wavelength $\lambda_{\mathrm{B}}=$ $1550 \mathrm{~nm}$, nonlinear refractive index $n_{2}=2.7 \times 10^{-17} \mathrm{~m}^{2} / \mathrm{W}$ and induced refractive index change $n_{g}=0.5 \times 10^{-4}$. In the Fig. 3, the curves $\mathrm{a}, \mathrm{b}$ and $\mathrm{c}$ represents the peak reflectivity of FBG at incident intensities of $1 \mathrm{MW} / \mathrm{cm}^{2}$ (Fig. 3 curve a), $50 \mathrm{MW} / \mathrm{cm}^{2}$ (Fig. 3 curve b) and $100 \mathrm{MW} / \mathrm{cm}^{2}$ (Fig. 3 curve c), respectively. One can also notice a few important features from Figure 3 such as (i) at an input intensity of $1 \mathrm{MW} / \mathrm{cm}^{2}$ (Figure 3(curve a)), the behaviour peak reflectivity of FBG is like a linear case. (ii) For input intensities of $50 \mathrm{MW} / \mathrm{cm}^{2}$ (Figure 3 (curve b)), the peak reflectivity at Bragg wavelength of grating is increases with length (L). (iii) At sufficiently high excitation intensity of $100 \mathrm{MW} / \mathrm{cm}^{2}$ (Fig. 3(curve c)), the peak reflectivity shows N-type like behavior. As the intensity of the input beam increases the stop band of the Bragg grating detunes itself towards the higher wavelength side from its original position (at $\lambda_{\mathrm{B}}$ ) because of the presence of the positive Kerr nonlinearity in the medium. If the input intensity is sufficiently high, the Bragg wavelength $\lambda_{B}$ is no longer lies within the stop band and the wavelength $\lambda>\lambda_{B}$ comes in the stop band as a result these wavelengths get more reflected. At this particular intensity the peak reflectivity reaches at $100 \%$ at the grating length $\mathrm{L}=8.5 \mathrm{~mm}$ and start to decrease after increasing the grating length. At grating length $\mathrm{L}=9.7 \mathrm{~mm}$ the reflectivity at Bragg wavelength becomes $0 \%$ and once again increases with increasing the grating length $(\mathrm{L})$. These nonlinear features of FBG can be exploited as tunable filter in lightwave communication system.

\section{CONCLUSION}

We have investigated the phenomenon of optical switching and optical transistor charaterstics in reflection mode of nonlinear fiber Bragg grating by incorporating Kerr effect in the coupled mode analysis. Also the peak reflectivity of the fiber Bragg grating at high excitation intensity is examined. To study optical limiting, we have compared the limiting action of FBG for four different incident wavelengths. The analysis shows that the switching action is dependent on the input intensity as well as on the wavelength of the incident light. In the case of optical transistor, the results predict that the transistor action is strongly dependent on the applied input intensity as well as on the operating wavelength of the incident light. Also we studied the effects of Kerr nonlinearity on the peak reflectivity of the FBG in detail. The results obtained have shown that the reflectivity of Bragg wavelength is increases with excitation intensity. According to these results, a FBG can be used as a tunable optical filter by precisely optimizing the grating length, the average refractive index deviation, and coupling strength of the grating.

\section{REFERENCES}

[1]. H. M. Gibbs, Optical Bistability: Controlling Light with Light, New York: Academic, 1985.

[2]. H. M. Gibbs, S. L. McCall and T. N. C. Venkatesan, "Differential gain and bistability using a sodium filled Fabry-Perot interferometer, Phys. Rev. Lett., 36 1135-1138 (1976).

[3]. N. G. R. Broderick, "Bistable Switching in Nonlinear Bragg Gratings," Optics Commun., 148, 90-94 (1998).

[4]. H. Lee and G. P. Agrawal, "Nonlinear switching of optical pulses in fiber Bragg grating,” IEEE J. Quantum Electronics, 39, 508-515 (2003).

[5]. S. Wabnitz, "Pulse self switching in optical fiber Bragg grating," Optics Communication, 114, 170-180 (1995).

[6]. B. J. Eggleton, R. E. Slusher, C. M. De Sterke, P. A. Krug and J. E. Sipe, "Bragg grating solitons," Phy. Rev. Lett., 76, 1627 (1996).

[7]. V. Janyani, J. D. Paul, A. Vukovic, T. M. Benson and P. Sewell, "TLM modelling of nonlinear optical effects in fiber Bragg grating," IEE Proc. Optoelectron., 151, 185-192 (2004).

[8]. H. G. Winful, J. H. Marburger and E. Garmire, Appl. Phys. Lett., 35, 379 (1979).

[9]. C. M. De Sterke and J. E. Sipe, "Switching dynamics of finite nonlinear media: A numerical study," Phy. Rev. A, 42, 2858-2869 (1990).

[10]. A. Melloni, M. Chinello and M. Martinelli, "All optical switching in phase shifted fiber Bragg grating," IEEE Photon. Technol. Lett., 12, 42-44 (2000).

[11]. D. Taverner, N. G. R. Broderick, D. J. Richardson, M. Ibsen and R. I. Laming, "All optical AND gate based on coupled gap-soliton formation in a fiber Bragg grating," Optics Letter, 23, 259-261 (1998).

[12]. Y. Yosia and Shum Ping, "Double optical bistability and its application in nonlinear chalcogenide-fiber Bragg grating," Physica B, 394, 293-296 (2007).

[13]. S. Pawar, S. Kumbhaj, P. Sen and P. K. Sen, "Fiber Bragg grating based intensity dependent optical notch filter," Nonlinear Optics Quantum Optics, 41, 253-264 (2010). 